\title{
Lattice rearrangement induced by excitons in cryocrystals
}

\author{
(C) E.V. Savchenko, A.N. Ogurtsov, O.N. Grigorashchenko
}

Verkin Institute for Low Temperature Physics \& Engineering, 310164 Kharkov, Ukraine

Experiments on permanent lattice defect formation induced by electronic excitation of Rare Gas Solids are presented. The creation of triplet excitons $\Gamma(3 / 2)$ is found to be the primary process, followed by the exciton self-trapping. Probable models of defect formation are discussed.

The self-trapping of excitons in atomic cryocrystals induces a variety of atomic processes including mass diffusion, and defect formation [1-4]. The basis for the physics of these phenomena is a concentration of the electronic excitation energy within a volume about that of a unit cell. Release of the energy can induce different kinds of lattice rearrangement. The above phenomena have been the subject of numerous experimental and theoretical studies on a variety of insulators [2,5-7]. Atomic cryocrystals with their simple lattice and well defined electronic structure [8] are excellent objects for investigation of exciton induced phenomena. The high quantum yield of these atomic processes caused by small binding energies in conjunction with a strong exciton-phonon interaction makes them especially suitable for experimental study.

A transformation of the initial point defects involving the self-trapped excitons was observed in solid $\mathrm{Ne}$ by the transient absorption method [9]. The creation of new lattice defects induced by the self-trapping of excitons in atomic cryocrystals was predicted in [10]. Vacuum ultraviolet spectroscopic study revealed the formation and accumulation of permanent lattice defects generated by excitation with slow electrons in atomic cryocrystals [4,11-17]. It was supposed that the self-trapping of excitons is the stimulating factor. The first direct evidence of the exciton's key role for the creation of radiation-induced defects was obtained in the experiments on selective excitation of solid $\mathrm{Xe}$ [18] and $\mathrm{Ar}$ [19] to the lowest exciton band $\Gamma(3 / 2), n=1$. This paper reviews experiments on permanent lattice defect formation induced by electronic transitions in atomic cryocrystals. As an example recent results on local lattice rearrangement induced by the excitation of atomic and molecular states of Ar are presented.

\section{Experiment}

Because a manifold of electronic levels can be involved in the phenomena, the optical spectroscopy is the most useful method for such kinds of investigations. The study was perfomed using cathodoluminescence, photoluminescence, and thermoluminescence methods. To reveal the effect of irradiation on luminescence spectra, it is necessary to grow the samples with the lowest possible concentration of initial defects and impurities. Suitable transparent samples were grown in special cryogenic cells under isobaric conditions $(P=80 \mathrm{~Pa})$ with a constant cooling rate of
$0.1 \mathrm{~K} / \mathrm{s}$. The sample quality was monitored by measuring the luminescence intensity distribution and with the glow curve analysis. Synchrotron radiation and a beam of electrons with low enough energy to avoid a knockon defect formation were used for the excitations of the samples. The dose dependence of the luminescence spectra was measured at low temperatures to minimize thermally activated processes. Stable point defects were detected by a sensibilized luminescence method. The defect component of the internal structure $[12,21]$ of the well known atomic and molecular bands [1] served as an indicator of lattice rearrangement. Thermoluminescence measurements were a complementary test of defect creation in the samples.

\section{Creation of defects}

It is well known $[1,3,8]$ that excitation of atomic cryocrystals from the ground state either by ionizing radiation or by photons results in self-trapping or trapping of excitons into atomic-type (A-STE, A-TE) and molecular-type (M-STE, $\mathrm{M}-\mathrm{TE})$ states. These states can be considered as short-lived defects which annihilate via radiative transititons. A part of them can convert to stable long-lived defects in the lattice. Since the energy of electronic excitation is transferred into kinetic energy of atomic motion over a unit cell, formation of three-, two, or one-dimensional defects is ruled out. In this case, only the point radiation defects, viz., Frenkel pairs (interstitials and vacancies) may emerge in the bulk of the crystal.

\section{Conversion from atomic-type states}

The analysis of the cathodoluminescence spectra of solid $\mathrm{Ne}$ [12] under different crystal-growth conditions as well as under subthreshold and superthreshold excitation made it possible to elucidate an origin of internal structure of the A-STE bands. It was shown that each band of the bulk emission associated with the transitions ${ }^{3} P_{1} \rightarrow{ }^{1} S_{0}$ and ${ }^{1} P_{1} \rightarrow{ }^{1} S_{0}$ consists of two components. The high-energy component stems from A-STE in a regular lattice, the low-energy one appears to be associated with structural defects. The emision spectrum of Ar atoms in solid $\mathrm{Ne}$ shows a similar structure (Fig. 1). A pronounced increase in the intensity of the defect component during irradiation by subthreshold energy electrons has been observed for both A-STE and A-TE in the Ne lattice. It was interpreted as being an outcome of a 


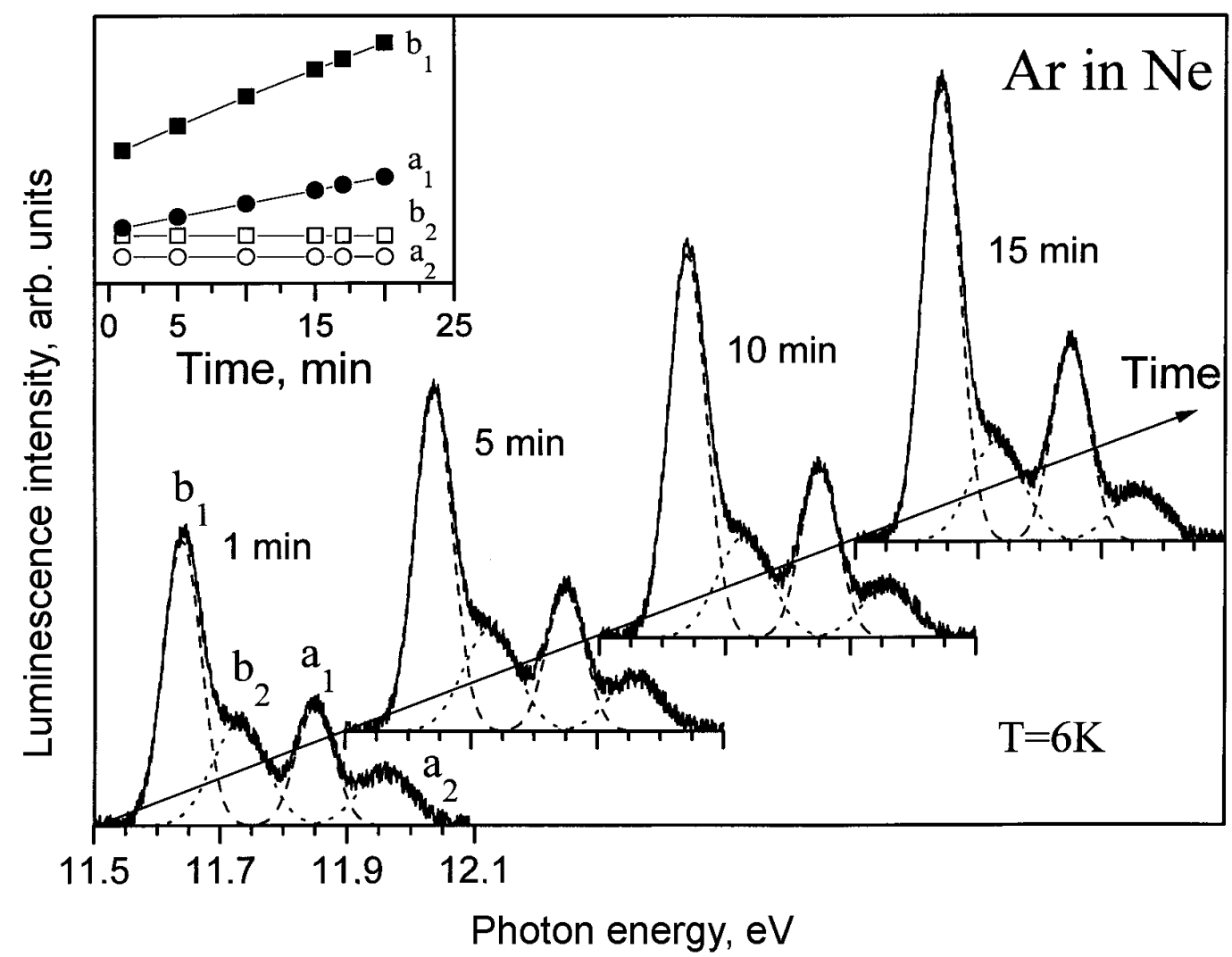

Figure 1. Modification of the atomic emission with exposure time. The insert shows the dose dependence of the component.

accumulation of permanent lattice defects generated during the lifetime of A-STE and A-TE. A current excited state molecular dynamics study [20] supports this interpretation and suggests a microscopic model of the conversion from A-STE to Frenkel pairs. The formation of the A-STE bubble with elastic lattice relaxation has been well studied earlier $[1,2]$. It is formed due to prevaling repulsive forces because of the negative electron affinity $(E=-1.2 \mathrm{eV}$ in solid $\mathrm{Ne}$ ). After the bubble formation the surrounding ground state atoms appear to have moved to the second shell. It was found that the second-nearest neighboring vacancyinterstitial pairs can create permanent defects which remain in the lattice after the exciton annihilation. The lowest energy bubble with plastic deformation can be considered as the primary STE-bubble accompanied with two nonadjacent vacancies in the first atomic shell and interstitials in the second one. The calculated transition energies from the defect-associated states of A-STE (54-66 meV) and A-TE $(105 \mathrm{meV})$ are in an excellent agreement with the experimental data $(63 \mathrm{meV}$ and $100 \mathrm{meV})$.

\section{Conversion from molecular-type states}

The two component internal structure of the STE luminescence bands appears to be a general feature of M-STE in atomic cryocrystals formed from heavier rare gases $[17,21]$ as well as for the considered A-STE states. The radiative decay of the self-trapped and trapped excitons in these solids produces the well known M-bands related to the ${ }^{1.3} \Sigma_{u}^{+} \rightarrow{ }^{1} \Sigma_{u}^{+}$transitions of molecular centers $\mathrm{Rg}_{2}^{*}$ ( $\mathrm{Rg}$ stands for a rare gas atom). The highenergy component of the M-band is supposed to stem from the exciton self-trapping in a regular lattice. The low-energy component is associated with exciton trapping in lattice imperfections. The internal structure of the M-band in solid $\mathrm{Ar}$ is shown in Fig. 2. The excitation spectra of the above components measered by the "redblue-side" subband separation method [18] demonstrate that the defect component can be excited below the bottom of the exciton band. An analysis of transformation of the M-band during irradiation by low-energy electron [16] has revealed a distinct intensity increase of the defect component with exposure time. The enhancement of the defect component upon a low-temperature irradiation of samples in subthreshold regime can be regarded as a proof of the stable defect formation induced by an excitation of the electron subsystem. An additional support of this conclusion has been inferred from the study of the glow curve (total intensite of the thermoluminescence) variation with exposure time [22]. It was assumed that the stimulating factor of the defect formation is a self-trapping of the triplet excitons into molecular type states. 


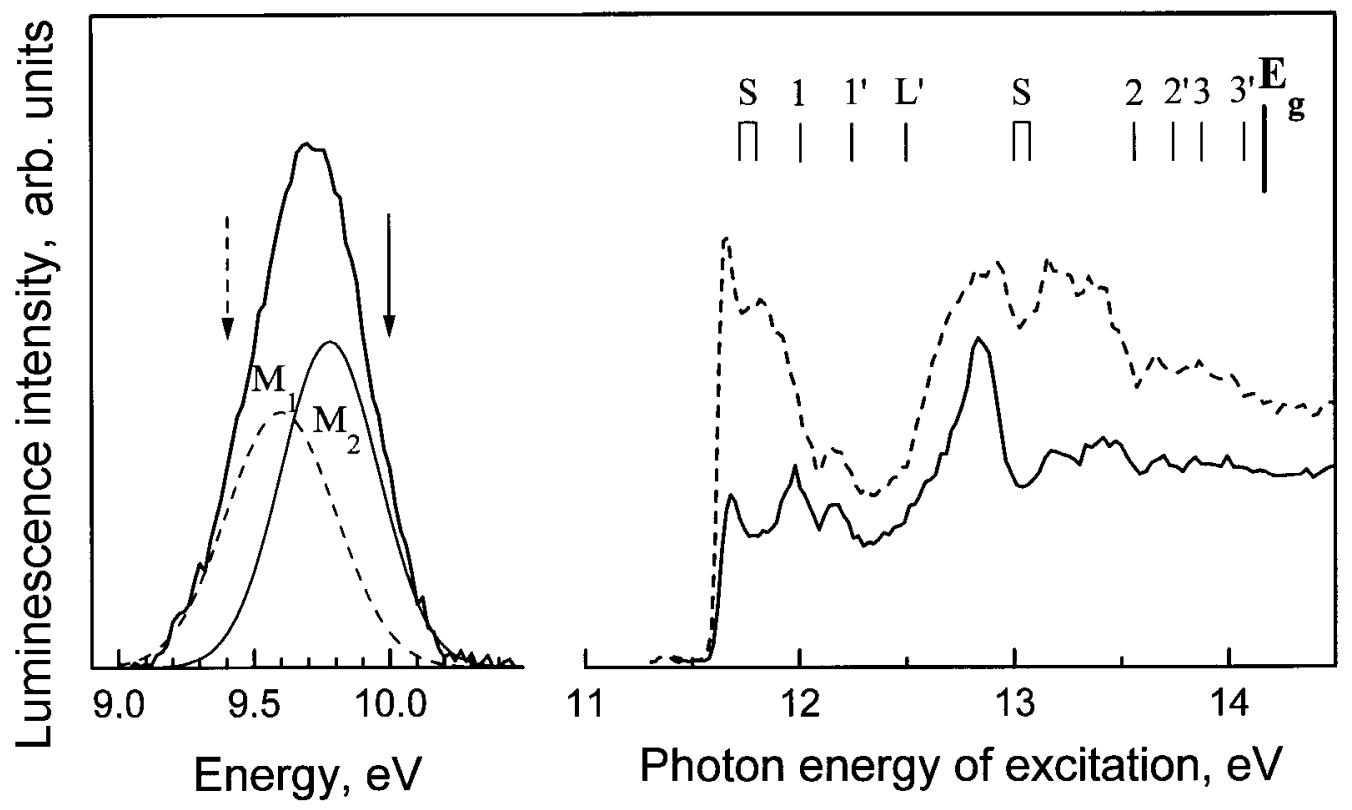

Figure 2. Internal structure of the molecular luminescence band of solid Ar. The arrows indicate the energies taken at the luminescence yield measurements [21].

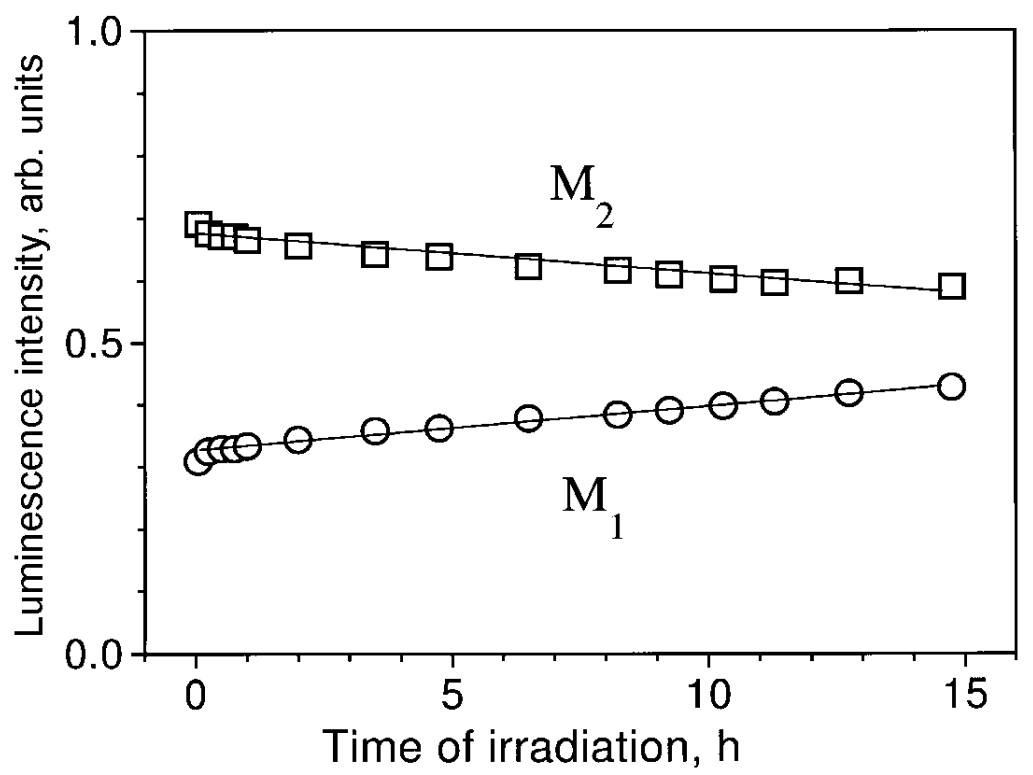

Figure 3. Dose dependence of the molecular luminescence component measured under selective photoexcitation at $T=7 \mathrm{~K}$.

The use of synchrotron radiation to investigate the defect formation in atomic cryocrystals has the advantage to excite definite electronic states and permits to reveal the primary states involved into the process. The excitation of solid $\mathrm{Ar}$ by photons with the energy in the range of the exciton band $\Gamma(3 / 2)$ results in an intensity redistribution of the M-band components in favor of the defect one (Fig. 3). The data obtained demonstrate the intrinsic nature of the phenomenon and confirm beyond any doubt the key role of excitons in the creation of radiation induced defects.
The newly-formed defects are thought to be Frenkel pairs. According to theoretical calculations [23] the only stable form of an intersitial atom in atomic cryocrystals is the split $\langle 100\rangle$ "dumb-bell" form. Taking this into account, the model of conversion from M-STE to stable lattice defects was proposed [4]. It was suggested that the defects are created and stabilized within the lifetime of self-trapped excitons. The process is supposed to occur by a displacement of the self-trapped exciton from the centrosymmetric position in the $\langle 110\rangle$ direction followed by a reorientation to $\langle 100\rangle$ 
direction to stabilize the defect. The redistribution of the component intensities associated with the self-trapped excitons in on-center and off-center positions seems to be in line with the assumption. Note that in the starting point of the exciton self-trapping the M-STE center is a system with a strong local vibration. In this case the energy release can proceed in a jumplike process [24]. If this energy release exceeds the energy needed for a local rearrangement of the lattice distroted by an electronic excitation, one can expect generation of defects. However the furher elucidation of the microscopic model invites to theoretical studies of the M-STE conversion to stable point defects.

\section{Ackowledgment}

The authors acknowledge fruitful discussions with Professors I.Ya. Fugol' and G.Zimmerer. We thank Professor K.S. Song for his interest in this work and making his results available prior to publication.

Financial support by the BMFT (grant 05650 GUB) and PECO-Project is gratefully acknowledged.

\section{References}

[1] I.Ya. Fugol'. Adv. Phys. 37, 1 (1988).

[2] K.S. Song, R.T. Williams. Self-Trapped Excitons. 2ed. SpringerVerlag (1996).

[3] G. Zimmerer. Nucl. Instrum. \& Meth. Phys. Res. B91, 601 (1994).

[4] E.V. Savchenko, A.N. Ogurtsov, O.N. Grigorashchenko, S.A. Gubin. Chem. Phys. 189, 415 (1994).

[5] N. Itoh, K. Tanimura. J. Phys. Chem. Sol. 51, 717 (1990).

[6] M.A. Elango. Elementary inelastic radiation-induced processes. American Institute of Physics, N.Y. (1991).

[7] Ch.B. Lushchik, A.Ch. Lushchik. Decady of electronic excitation with defect formation on solids. Nauka, M. (1989).

[8] N. Schwentner, E.-E. Koch, J. Jortner. Electronic Excitation in Condensed Rare Gases. Springer Tracts Mod. Phys. 60, (1986).

[9] T. Suemoto, H. Kanzaki. J. Phys. Soc. Jpn. 49, 1039 (1980).

[10] F.V. Kusmartsev, E.I. Rashba. Czech. J. Phys. 32, 54 (1982).

[11] E.V. Savchenko, Yu.I. Rybalko, I.Ya. Fugol'. JETP Lett. 42, 260 (1985).

[12] E.V. Savchenko, Yu.I. Rybalko, I.Ya. Fugol'. Sov. J. Low Temp. Phys. 14, 220 (1988).

[13] I.Ya. Fugol', A.N. Ogurtsov, O.N. Grigorashchenko, E.V. Savchenko. Sov. J. Low Temp. Phys. 18, 27 (1992).

[14] E.V. Savchenko. J. Electron Spectrosc. Relat. Phenom. 79, 47 (1996).

[15] I.Ya. Fugol, E.V. Savchenko, A.N. Ogurtsov, O.N. Grigorashchenko. Physica B190, 347 (1993).

[16] E.V. Savchenko, A.N. Ogurtsov, O.N. Grigorashchenko, S.A. Gubin. Low Temp. Phys. 22, 926 (1996).

[17] E.V. Savchenko, A.N. Ogurtsov, S.A. Gubin, O.N. Grigorashchenko. J. Lumin. 72-74, 711 (1997).

[18] D. Varding, J. Becker, L. Frankensten, B. Peters, M. Runne, A. Schröder, G. Zimmerer. Low Temp. Phys. 19, 427 (1993).
[19] J. Becker, A.N. Ogurtsov, M. Runne, E.V. Savchenko, G. Zimmerer. In: HASYLAB Annial Report 1996. DESY, Hamburg (1997). $251 \mathrm{p}$.

[20] Chun-rong Fu, K.S. Song. J. Phys. Condens. Matter (accepted for publication).

[21] A.N. Ogurtsov, E.V. Savchenko, S.A. Gubin, O.N. Grigorashchenko, M. Runne, A. Karl, J. Becker, G. Zimmerer. In: Excitonic Processes in Condensed Matter / Ed. M. Schreiber. Dresden University Press, Dresden (1996). P. 195.

[22] A.N. Ogurtsov, E.V. Savchenko, O.N. Grigorashchenko, S.A. Gubin, I.Ya. Fugol'. Low Temp. Phys. 22, 922 (1996).

[23] R.M.J. Cotteril, M. Doyama. Phys. Lett. A25, 35 (1967).

[24] V. Hizhnyakov. Phys. Rev. B53, 13981 (1996). 